\title{
Validating the Farsi version of the Pregnancy Worries and Stress Questionnaire (PWSQ): An exploratory factor analysis
}

Fariba Navidpour ${ }^{1}$, Mahrokh Dolatian $^{2}$, Sara Shishehgar ${ }^{3}$, Farideh Yaghmaei $^{4}$, Hamid Alavi Majd ${ }^{5}$, Seyed Saeed Hashemi $^{6}$

${ }^{1}$ M.Sc. of Midwifery, Department of Midwifery and Reproductive Health, School of Nursing and Midwifery, Shahid Beheshti University of Medical Sciences, Tehran, Iran

${ }^{2}$ Ph.D. of Social Determinants of Health, Assistant Professor, Department of Midwifery and Reproductive Health, School of Nursing and Midwifery, Shahid Beheshti University of Medical Sciences, Tehran, Iran

${ }^{3} \mathrm{Ph} . \mathrm{D}$. Candidate, Faculty of Health, University of Technology, Sydney, Australia

${ }^{4}$ Ph.D. in Public Health, Associate Professor, Department of Nursing, School of Nursing and Midwifery, Zanjan Branch, Islamic Azad University, Zanjan, Iran

${ }^{5}$ Ph.D. in Biostatistics, Department of Biostatistics, School of Paramedical Sciences, Shahid Beheshti University of Medical Sciences, Tehran, Iran

${ }^{6}$ Ph.D. in Psychiatrics, Department of Psychology, Tehran University, Tehran, Iran

\section{Type of article: Original}

\begin{abstract}
Introduction: Biological, environmental, inter- and intrapersonal changes during the antenatal period can result in anxiety and stress in pregnant women. It is pivotal to identify potential stressors and prevent their foetal and maternal consequences. The present study was conducted to validate and examine the factor structure of the Farsi version of the Pregnancy Worries and Stress Questionnaire (PWSQ).

Methods: In 2015, 502 Iranian healthy pregnant women, referred to selected hospitals in Tehran for prenatal care at 8-39 weeks of pregnancy, were recruited through a randomized cluster sampling. The PWSQ was translated into Farsi, and its validity and reliability were examined using exploratory factor analysis by SPSS version 21 .

Results: The content validity of items on the PWSQ was between 0.63-1. The content validity index for relevance, clarity and simplicity were $0.92,0.98$, and 0.98 , respectively, with a mean of 0.94 . The Kaiser-MeyerOlkin measure of sampling adequacy was 0.863 . Test-retest reliability showed high internal consistency $(\alpha=0.89$; $\mathrm{p}<0.0001)$

Conclusion: The psychometric evaluation and exploratory factor analysis showed that the translated questionnaire is a valid and reliable tool to identify stress in Iranian pregnant women. Application of the questionnaire can facilitate the diagnosis of stress in pregnant women and assist health care providers in providing timely support and minimizing negative outcomes of stress and anxiety in pregnant women and their infants.
\end{abstract}

Keywords: Exploratory examination; Factor analysis; Pregnancy; anxiety; Stress; Worry

\section{Introduction}

Pregnancy is expected to be a joyful experience in every woman's life. This natural process can, however, be disturbed by a variety of factors and put tension and strain on pregnant women and their families (1). These factors include, but are not limited to, physical, physiological and psychosocial alterations, inter- and intrapersonal changes, financial matters, and pregnancy-related medical complications and concerns about newborn's well-being and childbirth procedure (2). Tension is defined as a condition in which an individual's normal psychological function is

\section{Corresponding author:}

Assistant Professor Dr. Mahrokh Dolatian, Department of Midwifery and Reproductive Health, School of Nursing and Midwifery, Shahid Beheshti University of Medical Sciences, Tehran, Iran.

Tel: +98.2188202517, Fax: +98.2188202517, Email: mhdolatian@gmail.com

Received: June 09, 2016, Accepted: September 07, 2016, Published: October 2016

iThenticate screening: September 02, 2016, English editing: September 20, 2016, Quality control: October 08, 2016

(C) 2016 The Authors. This is an open access article under the terms of the Creative Commons Attribution-NonCommercialNoDerivs License, which permits use and distribution in any medium, provided the original work is properly cited, the use is non-commercial and no modifications or adaptations are made. 
impaired by internal and external factors. This can lead to the stimulation of autonomic nervous system, especially sympathetic system, followed by anxiety and stress $(2,3)$. The World Health Organization (WHO) (4) puts forward many social and cultural concerns within the framework of pregnancy tensions and worries, some of which relate to the postpartum period. Having many children, infant's gender, and not being in a good relationship with the partner as a result of problems that arise during and after pregnancy are among issues recognised by the WHO as having an influence on emergence of pregnancy tensions. Tension also can result in the stimulation of hypothalamus-pituitaryadrenal (HPA) axis causing an increase in cortisol secretion from the cortex of adrenal gland. The increase of cortisol is followed by negative feedback on the HPA axis and adrenocorticotropic hormone (ACTH). Other unwanted physiological changes of tension are the release of epinephrine and nor-epinephrine due to the stimulation of adrenal medulla. A combination of the aforementioned physiological changes contribute to anxiety, which is defined as an unpleasant sense of fear and worry accompanied by physical symptoms such as headache, palpitation, stomach ache, shortness of breath, sweating, agitation, and pallor $(2,3)$. The stimulation of endocrine system also may lead to more severe outcomes such as severe hyperemesis gravidarum, preterm labour, low birth weight, fetus growth restriction, poor neonatal outcomes, worsening of primary heart, coronary diseases, and high blood pressure (5). Different studies have reported the prevalence of antenatal tension to vary between $16.7 \%$ and $74 \%$ during the first trimester of pregnancy $(6,7)$. In particular, two Iranian studies demonstrated that $16.7 \%$ and $49 \%$ of the women suffered tension and anxiety, respectively, during pregnancy $(7,8)$. With regards to the importance of tension and its negative impacts on mothers and newborns, a valid and reliable questionnaire is required to effectively identify stressors during pregnancy and help prevent the adverse events. To this aim, various studies have utilized different tools and measures to investigate the level of tension and stress during pregnancy. For example, in a study by Arch et al., the Pregnancy Worries and Stress Questionnaire (PWSQ) was used to examine the tendency of pregnant women American toward alcohol consumption (9). The PWSQ is a shortened combination of the Pregnancy-Related Anxiety Questionnaire (PRAQ) and Van Den Bergh's questionnaire and used to identify pregnancy-related stressors (10). This tool has been translated into different languages and has shown high levels of validity and reliability. Because the PWSQ is a standard tool and because, to the best knowledge of the author, there was no standardised tool in Farsi to assess antenatal stress, this study was conducted to examine the validity and factor structure of the Farsi version of the PWSQ to be used in the population of Iranian pregnant women.

\section{Material and Methods}

\subsection{Research design and participants}

The target population for the study was pregnant women referred to prenatal care units of four selected hospitals in Tehran, Iran, in 2015. Women were considered to participate in the study if they met the following inclusion criteria: (a) age between 18 and 45 years old; (b) gestational age between 8 and 39 weeks; (c) no experience of stressful events during the past six months; (d) not having either a physically or mentally impaired child; (e) being pregnant with a singleton foetus; and (f) living with their husband at the time of participating in the study. Women who did not meet one or more of the inclusion criteria were excluded from the study.

\subsection{Sample size}

Sample size was calculated based on the number of the questions in the questionnaire so as to help the researchers investigate the validity, in particular construct validity, and reliability of the questionnaire. Based on power calculation $(80 \%)$ and in order to allow a potential drop-out rate of $10 \%, 550$ pregnant women were required to participate in the study $(11,12)$.

\subsection{Measurement tools}

Data collection was carried out using the Pregnancy Worries and Stress Questionnaire (PWSQ) as well as demographics and obstetric questionnaire. The PWSQ contains 25 items and includes six subcategories as follows: mother's health (six items), childbirth and mother's experience (four items), newborn's health (five items), personalfamily (five items), personal-occupational (three items), and mother-newborn bonding (two items). Each item in the 5-point scale was answered from 0 for never to 4 for always. Sum of the scores, then, was rated between $0-100$. The implemented obstetric and demographic questionnaire contained five sections and 36 questions. The questionnaire did not need to be assessed in terms of validity and reliability. The standard "forward-backward" procedure (13) was applied to translate the questionnaire from English to Farsi. Two independent bilinguals translated the 25 items into Farsi and, then, the preliminary version was back translated into English. In order to maximize cultural adaptation and create a version as conceptually close to the original questionnaire as possible, the Persian version of PWSQ-25 was reviewed by a panel of experts. The experts were able to identify unclear or vague questions and comment on problematic items. The final version of the questionnaire was approved by the panel of experts. 


\subsection{Procedure}

Participants were recruited using random cluster sampling as follows. Firstly, Tehran, the capital of Iran, was divided into five zones: north, south, west, east, and central. The target hospitals were then identified in each zone. Next, one or two hospitals were randomly selected from each zone; finally, 502 pregnant women referred to the randomly selected hospitals and met the inclusion criteria were invited to participate in the study $(11,12)$. They were given an information sheet and were provided enough time to read it and ask their questions about the study. Those who were interested in participating in the study provided their written consent and completed the study questionnaires (14).

\subsection{Data analysis}

The collected data were analyzed using Statistical Package for Social Science, Advanced Statistics, Release 21.0 (SPSS for windows, SPSS Inc., Chicago, IL, USA). A panel of experts examined the content, face, and criterion validity of the questionnaire. A quantitative approach was adopted to examine content validity, content validity ratio (CVR), and content validity index (CVI). The content validity ratio was examined by 13 experts who were requested to score each item based on a 3-degree spectrum, which included "necessary," "useful but not necessary," and "not necessary" $(15,16)$. To examine the content validity index, the same experts were requested to specify each item's "relevance," "simplicity," and "clarity" based on Walts and Bausell index (17). Criterion validity was examined using the Speilburger's state-trait anxiety inventory (STAI) (18). Moreover, exploratory factor analysis (EFA) was used to validate the construct of the questionnaire. In EFA some items were removed or abridged. Adequacy of the sample size was assessed using Kaiser-Meyer-Olkin's measure of sampling adequacy and the Bartlett's test of sphericity prior to factor analysis. To determine the number of the questionnaire components, eigenvalue and scree plot were used. Then, the components of the PWSQ were extracted through principle component analysis as well as Varimax rotation. Because it was the first time the questionnaire was used in Iran, the factor loading was considered less than 0.3. In terms of examining the questionnaire reliability, internal consistency and temporal stability were assessed and presented by Cronbach alpha and test-retest, respectively (19).

\subsection{Ethics of research}

The Human Research Ethics Committee (HREC) in Shahid Beheshti Medical University, Tehran Medical University, Social Security Organization, and the hospitals' management organizations approved the protocol prior to the commencement of study. The researchers obtained written permission from the designers of PWSQ to translate it into Farsi and explore factor analysis of the Farsi version. Prior to the study, the participants were informed about the study aims, and they were assured about confidentiality.

\section{Results}

In this research, 502 out of 550 participants completed the survey. Table 1 shows the participants' demographic characteristics. In terms of face validity, all 25 items of the PWSQ were confirmed. Because the impact score was higher than 1.5 in the quantitative sector, no item was removed. In terms of qualitative content validity, the items were specified by experts. In quantitative content validity, the validity of the items was calculated between 0.63 and 1. No item was eliminated because, according to the Lawshe, minimum acceptable CVR value should be 0.54 . Regarding CVI, relevance (0.92), clarity (0.98), and simplicity (0.98) of each item, as well as the average of the three characters, $(0.92)$ were assessed. According to the results, no item was eliminated in this stage. In terms of criterion validity, correlation between total score of the PWSQ and scores of the participants' hidden and apparent anxiety was examined in two separate stages $(\mathrm{r}=0.707, \mathrm{p}=0.000 ; \mathrm{r}=0.692, \mathrm{p}=0.000)$. In addition, correlation between total score of the PWSQ and the STAI was calculated $(\mathrm{r}=0.725, \mathrm{p}=0.000)$. According to the KMO, adequacy of samples was 0.86 . Because the KMO ranges between 0 and 1, the higher score guarantees a more reliable EFA. Furthermore, the Bartlett's test of sphericity demonstrated a significant difference in correlation matrix $(\mathrm{p}=0.000)$ (Table 1). According to the results of the KMO and the Bartlett's test, factor analysis was validated. The varimax rotation matrix also was carried out (the results of which are presented in Table 2). Table 3 clearly demonstrates that the eigenvalue of the six categories in the PWSQ are higher than 1 . These six categories, generally, present $60.1 \%$ of the variance. Total internal consistency of the PWSQ was examined with 50 participants $(\alpha=0.89)$. Internal consistency also was calculated for each category. Accordingly, Cronbach's alpha for category 1 to 6 was $0.82,0.79$, $0.71,0.75,0.71$, and 0.73 , respectively. To examine the reliability of the questionnaire, test-retest was used with a two-week interval on 30 pregnant women $(\mathrm{p}=0.000, \mathrm{r}=0.98)$. 
Table 1. KMO Measure of Sampling Adequacy and Bartlettt's Test of Sphericity Bartlettt's Test of Sphericity and Measure of Sampling Adequacy

\begin{tabular}{|l|l|l|}
\hline KMO measure of sampling adequacy & 0.863 \\
\hline Bartlettt's test of sphericity & Approx. chi-square & 356.479 \\
\cline { 2 - 3 } & $\mathrm{df}$ & 300 \\
\cline { 2 - 3 } & $P$-value & 0.0001 \\
\hline
\end{tabular}

$\mathrm{df}=$ Degree of Freedom

Table 2. Factor Analysis (Varimax Rotation) of the Pregnancy Worry and Stress Questionnaire

\begin{tabular}{|l|l|l|l|l|l|}
\hline Factor Analysis (Varimax Rotation) of the Pregnancy Worry and Stress Questionnaire \\
\hline
\end{tabular}

Table 3. Factor Analysis of the Six Subclasses of PWSQ

\begin{tabular}{|l|l|l|l|}
\hline Factors & Equity & Variance & Cumulative Variance \\
\hline 1.Mother's Health & 7.213 & 28.851 & 28.851 \\
\hline 2.Newborn's Health & 2.005 & 8.019 & 36.870 \\
\hline 3.Personal family & 1.899 & 7.596 & 44.466 \\
\hline 4.Childbirth and maternal experience & 1.560 & 6.240 & 50.706 \\
\hline 5. Personal occupational & 1.234 & 4.938 & 55.644 \\
\hline 6. Mother-newborn bonding & 1.114 & 4.457 & 60.101 \\
\hline
\end{tabular}




\section{Discussion}

Uncontrolled worry, stress, and anxiety threaten a mother and newborn's health. Recognising worried women and counselling them can mitigate the adverse effects of worry and stress on pregnant mothers. To identify the stressors, a highly valid and reliable tool is of utmost importance (20). The results of the current study confirmed the adequacy, reliability and feasibility of the PWSQ. According to the exploratory analysis, the six categories of the questionnaire were matched with the Arch et al.'s questionnaire. On the other hand, Huizink et al. (21) carried out a study on Dutch pregnant women. They used the 34-item pregnancy-related anxiety questionnaire, which include questions about fear of a shift in relations within partners, fear of changes in mood and communication, and maternal love. They did not find any acceptable comparative fit index, which was accompanied with a variance with a high error. The remarkable point in Arch et al.'s study is that $49 \%$ of mothers' worries was related to the family income and parity. In this study, while financial issues were confirmed as an effective factor on pregnant women's worry, parity did not turn out to be significant. Reliability is a significant criterion of quality of a scale. In this study, Cronbach's alpha coefficient and test-retest confirmed the reliability, internal consistency, and stability of the PWSQ. A reliable scale can increase a study's power in detection of differences (20). According to the results of this study, there is a significant relationship between the items of the PWSQ and the whole scale. Accordingly, worry and stress of pregnancy have a significant relationship with worrying about childbirth, newborn's health, mother's health, personal family factors, and personal occupational factors. In addition, Arch et al., using a regression model, found a relationship between socio-family factors and pregnancy-related worry. The current study has two important features. First, this study considered all psychometric and standardization factors of the PWSQ. In previous studies, there is a scarcity in detection of pregnancy-related stress using a questionnaire validated with adequate sample size. Considering the theoretical models in designing as well as standardization of questionnaires increases quality and reliability of the studies. Consequently, the results of such studies can recommend reliable interventions to decrease pregnancy-related stress (22). The second feature is related to the research participants. Participants in this study consisted of all pregnant women from various socio-economic levels and residential areas in Tehran, the capital of Iran. The parity of the participants was not of importance as both multiparous and nulliparous women entered the study in the same conditions. However these features were similar to Arch et al.'s study, while being inconsistent with those of Huizink et al.'s study.

\section{Conclusions}

The current study's aim was to explore the validity and reliability of the pregnancy worry and stress questionnaire. According to the results, the questionnaire suits Iranian pregnant women as a vulnerable population. Safety and satisfaction of pregnant women are the optimum purpose of health providers as well as all caring individuals. In addition, underestimating stressors may result in adverse outcomes in mothers and newborns while timely recognition of distressed mothers and helping them overcome the stressors can contribute to a safe pregnancy and childbirth progress. Using an appropriate scale by health providers, in particular midwives and obstetricians, may result in identifying the stressors and providing a broad spectrum of services such as counseling and training sessions for pregnant women. These services would decrease concerns and stresses and increase a mother's capacity for childbirth. With this aim in view, timely pregnancy and childbirth progress training sessions may result in lessening concerns and worries in mothers. Moreover, providing mothers with appropriate diet and exercise can assure them about their weight gain and appearance. Furthermore, the appropriate screening medical tests can decrease a mother's concerns about fetal health. To conclude, the examined PWSQ is recommended to all prenatal care units, midwives, and obstetricians as well as medical, midwifery, nursing, and psychology students throughout Iran.

\section{Acknowledgments:}

This study is a part of a dissertation of a master's degree with record number 461/M/P/91. We, the authors, wish to thank Joanna J. Arch, member of the psychology department in Colorado University.

\section{Conflict of Interest:}

There is no conflict of interest to be declared.

Authors' contributions:

All authors contributed to this project and article equally. All authors read and approved the final manuscript. 


\section{References:}

1) Cha S, Masho SW. Preterm birth and stressful life events. In O Erez (Ed.), Preterm birth. InTech . 2013. doi: $10.5772 / 54978$.

2) Sadock BJ, Sadock VA. Kaplan \& Sadock's concise textbook of clinical psychiatry. 2008: Lippincott Williams \& Wilkins.

3) Tordjman S, Gragnier-Deferre C, Graignic R. Ensations during pregnancyand Life Events Questionnaire. Hogrefe. 2014.

4) WHO. Maternal mental health and child health and development in low and middle income countries. report of the meeting, Geneva, Switzerland, 30 January-1 February, 2008. 2008.

5) Sandman CA, Davis EP, Glynn LM. Psychobiological stress and preterm birth. 2012: INTECH Open Access Publisher.

6) Rubertsson C, Hellström J, Cross M, Sydsjö G. Anxiety in early pregnancy: prevalence and contributing factors. Arch Womens Ment Health. 2014; 17(3): 221-8. doi: 10.1007/s00737-013-0409-0. PMID: 24442712.

7) Salari P, Firoozi M, Sahebi A. Study of the Stressors Associated with Pregnancy. Journal of Sabzevar University of Medical Sciences. 2005; 12(3(37)): 34-40.

8) Alipour Z, Lamyian M, Hajizadeh E. Anxiety and fear of childbirth as predictors of postnatal depression in nulliparous women. Women Birth. 2012; 25(3): e37-43. doi: 10.1016/j.wombi.2011.09.002. PMID: 21959041.

9) Arch JJ. Pregnancy-specific anxiety: which women are highest and what are the alcohol-related risks? Compr Psychiatry. 2013; 54(3): 217-28. doi: 10.1016/j.comppsych.2012.07.010. PMID: 22943960.

10) Bea Van den Bergh. The influence of maternal emotions during pregnancy on fetal and neonatal behavior. 1990; 5(2): 119-30.

11) Schneider Z. Nursing and midwifery research: Methods and appraisal for evidence-based practice. 2013: Elsevier Australia.

12) Munro BH. Statistical methods for health care research. 2013, Philadelphia: Lippicott. Williams \& Wilkins. 557.

13) Acquadro C, Lafortune L, Mear I. Quality of life in multiple sclerosis: translation in French Canadian of the MSQoL-54. Health Qual Life Outcomes. 2003; 1: 70. doi: 10.1186/1477-7525-1-70. PMID: 14636427, PMCID: PMC317365.

14) Leinonen T, Leino-Kilpi H, Stahlberg MR, Lertola K. The quality of prospective care: Development of a tool for the perceptions of patients. J Adv Nurs. 2001; 35(2): 294-306. doi: 10.1046/j.13652648.2001.01846.x. PMID: 11442708.

15) Polit DF, Beck CT, Hungler B. Essentials of nursing research: Methods, Appraisal, and Utilization. 2006, Lippincott Williams \& Wilkins: Philadelphia.

16) Polit DF, Beck CT, Owen SV. Is the CVI an acceptable indicator of content validity? Appraisal and recommendations. Res Nurs Health. 2007; 30(4): 459-67. doi: 10.1002/nur.20199. PMID: 17654487.

17) Waltz C, Bausell B. Nursing Research: Design, Statistics and Computer Analysis FA. 1983, Philadelphia: Davis Company.

18) Spielberger CD, Gorsuch RL, Lushene RE. Manual for the state-trait anxiety inventory. 1970.

19) Polit DF, Beck CT. Essentials of nursing research: Appraising evidence for nursing practice. 2013: Lippincott Williams \& Wilkins.

20) Burns N, Grove SK. The practice of nursing research. 1993. WB Saunders Company.

21) Huizink AC, Mulder EJ, Robles de Medina PG, Visser GH, Buitelaar JK. Is pregnancy anxiety a distinctive syndrome? Early Hum Dev. 2004; 79(2): 81-91. doi: 10.1016/j.earlhumdev.2004.04.014. PMID: 15324989.

22) Alderdice F, Lynn F, Lobel M. A review and psychometric evaluation of pregnancy-specific stress measures. J Psychosom Obstet Gynaecol. 2012; 33(2): 62-77. doi: 10.3109/0167482X.2012.673040. PMID: 22554138. 